\title{
A Novel Approach for Solving Fuzzy Differential Equations Using Cubic Spline Method
}

\author{
S. Karpagappriya $\mathbb{D}^{1},{ }^{1}$ Nazek Alessa $\mathbb{D}^{\circ},{ }^{2}$ P. Jayaraman, ${ }^{1}$ and K. Loganathan $\mathbb{D}^{3}$ \\ ${ }^{1}$ Department of Mathematics, Bharathiar University, Coimbatore-641 046, Tamilnadu, India \\ ${ }^{2}$ Department of Mathematical Sciences, Faculty of Science, Princess Nourah Bint Abdulrahman University, Riyadh, Saudi Arabia \\ ${ }^{3}$ Research and Development Wing, Live4Research, Tiruppur, Tamilnadu 638 106, India
}

Correspondence should be addressed to K. Loganathan; loganathankaruppusamy304@gmail.com

Received 5 February 2021; Accepted 2 May 2021; Published 22 May 2021

Academic Editor: José Francisco Gómez Aguilar

Copyright (C) 2021 S. Karpagappriya et al. This is an open access article distributed under the Creative Commons Attribution License, which permits unrestricted use, distribution, and reproduction in any medium, provided the original work is properly cited.

\begin{abstract}
Ambiguity in real-world problems can be modeled into fuzzy differential equations. The main objective of this work is to introduce a new class of cubic spline function approach to solve fuzzy initial value problems efficiently. Further, the convergence of this method is shown. As it is a single-step method that converges faster, the complexity of the proposed method is too low. Finally, a numerical example is illustrated in order to validate the effectiveness and feasibility of the proposed method, and the results are compared with the exact as well as Taylor's method of order two.
\end{abstract}

\section{Introduction}

The entire real world is complex; it is found that the complexity arises from uncertainty in the form of ambiguity. Uncertainties in the real-world problem can be modeled easily with the help of fuzzy set theory when one lacks complete information about the variables and parameters [1]. This concept of fuzzy set theory was first introduced by Zadeh [2] in 1965. Chang and Zadeh explicated the concept of fuzzy derivatives [3]. The term fuzzy differential equation was formulated by Kandal and Byatt [4] in 1978. These equations help in modeling the propagation of epistemic uncertainty in a dynamical environment [5]. Kaleva [6], Seikkala [7], and Song and Wu [8] have extensively studied the existence and uniqueness of solutions of these equations. A general formulation of the first-order fuzzy initial value problem was given by Buckley and Feuring [9]. Later, the fuzzy initial and boundary value differential equation was given by O'Regan et al. [10].

First-order linear fuzzy differential equations have inspired several authors to focus on solving them numerically since they appear in many real-world applications. These applications include different fields of science such as medical diagnosis, biology, and civil engineering and also in the field of economics [11] where the information are not given in the crisp set [12]. Based on Zadeh's extension principle, a new fuzzy version of Euler's method was developed by Ahamed and Hasan [13]. Solving of these equations by the Taylor method of order $p$ has been studied by Abbasbandy and Viranloo [14], and the same was discussed by Allahviranloo et al. [15] by using the predictor-corrector algorithm. Finally, the authors concluded that a fuzzy differential equation can be modified into a system of ordinary differential equations (ODEs). Also, they found out that there are two solutions for a fuzzy differential equation by solving the associated ODEs. The convergence, consistency, and stability for approximating the solution of fuzzy differential equations with initial value conditions have been studied by Ezzati et al. [16]. All the numerical results of these equations and their applications were summarized by Chakraverty et al. [12].

In this paper, the fuzzy initial value problem is solved numerically by using a new class of function approximation called cubic spline, for better accuracy of the solution. 


\section{Preliminaries}

Let $X^{\prime}=\{x\}$ where $X^{\prime}$ is the space of points and $x$ is the generic element of $X^{\prime}$.

Definition 1 (see [2]). A fuzzy subset $\mu_{A^{\prime}}$ of the set $A^{\prime}$ in $X^{\prime}$ is a function $\mu_{A^{\prime}}: A^{\prime} \longrightarrow[0,1]$.

Definition 2 (see [17]). The $\alpha$-level set of the fuzzy set $A^{\prime}$ of $X^{\prime}$ is a crisp set $\left[A^{\prime}\right]^{\alpha}=\left\{x \in X^{\prime} \mid \mu_{A^{\prime}}(x) \geq \alpha\right\}$ if $\alpha \in(0,1]$.

Definition 3 (see [17]). Let $A^{\prime}$ be a triangular fuzzy number (TFN) which is defined as $\langle l, m, n\rangle$ where $[l, n]$ is the support, $\{m\}$ is the core, and the membership function is

$$
\mu_{A^{\prime}}(x)=\left\{\begin{array}{cll}
\frac{x-l}{m-l}, & \text { if } & x \in[l, m], \\
\frac{n-x}{n-m}, & \text { if } & x \in(m, n], \\
0, & \text { if } & x \notin[l, n],
\end{array}\right.
$$

where $l<m<n$.

Let us denote the set of all fuzzy numbers on $\mathbb{R}$ as $\mathbb{F}$ which is a fuzzy number such that $\mu: \mathbb{R} \longrightarrow[0,1]$.

Definition 4 (see [18]). Let $l$ and $m \in \mathbb{F}$. If there exists $n \in \mathbb{F}$ such that $l=m+n$, then $n$ is the Hukuhara difference of $l$ and $m$. This can be denoted as $n=l \ominus m$. To define the differentiability of a fuzzy function, we can make use of this difference as follows.

Let $H:[u, v] \longrightarrow \mathbb{F}$ be differentiable at $t_{0} \in(u, v)$. If there exists some element $H^{\prime}\left(t_{0}\right) \in \mathbb{F}$ such that

$$
\begin{aligned}
\lim _{h \longrightarrow 0^{+}} \frac{H\left(t_{0}+h\right) \ominus H\left(t_{0}\right)}{h} & =\lim _{h \longrightarrow 0^{+}} \frac{H\left(t_{0}\right) \ominus H\left(t_{0}-h\right)}{h} \\
& =H^{\prime}\left(t_{0}\right),
\end{aligned}
$$

then $H$ is said to be Hukuhara differentiable at $t_{0}$.

Suppose $H$ is differential at the point $t_{0} \in(u, v)$, then all its $\alpha$-level sets, $H_{\alpha}(t)=[H(t)]^{\alpha}$, are Hukuhara differentiable at $t_{0}$ and $\left[H^{\prime}\left(t_{0}\right)\right]^{\alpha}=\mathrm{DH}_{\alpha}\left(t_{0}\right)$, where $\mathrm{DH}_{\alpha}$ denotes the Hukuhara derivatives of $H_{\alpha}$ and $H_{\alpha}$ as the multivalued mapping.

Theorem 1 (see [19]). Let $q(x) \in C^{3}[u, v]$ and $\left(\Delta_{k}\right)$ be a sequence of partitions on $[u, v]$, with $\lim _{k \rightarrow \infty}\left\|\Delta_{k}\right\|=0$; then, for the interpolate cubic spline $S_{\Delta_{k}}(x)$, uniformly for $u \leq x \leq v$,

$$
\left|q^{(p)}(x)-S_{\Delta_{k}}^{(p)}(x)\right|=O\left(\left\|\Delta_{k}\right\|\right)^{3-p}, \quad \text { for } p=0,1,2 \text {, and } 3 \text {. }
$$

If $q^{\prime \prime \prime}(x)$ satisfies the Holder condition on $[u, v]$ with $0<\alpha<1$, then

$$
\left|y^{(p)}(x)-S_{\Delta_{k}}^{(p)}(x)\right|=O\left(\left\|\Delta_{k}\right\|\right)^{3+\alpha-p} .
$$

Proof. This theorem has been proved in the work by Ahlberg et al. [19] (p. 29).

2.1. Cubic Spline Function Approximation for Initial Value Problems. Let the given $(n+1)$ data points be $\left(u_{i}, v_{i}\right)$, $i=0,1,2, \ldots, n$, where $u_{0}<u_{1}<u_{2}<\ldots<u_{n}$. Let us define the cubic spline $P_{i}(u)$, which is defined in the interval $\left[u_{i-1}, u_{i}\right]$ as follows.

(i) For $u<u_{0}$ and $u>u_{n}, P_{i}(u)$ is a polynomial whose degree is one

(ii) $P_{i}(u)$ is at most a cubic polynomial in each subinterval $\left[u_{i-1}, u_{i}\right]$, where $i=1,2, \ldots, n$

(iii) $P_{i}(u), P_{i}^{\prime}(x)$, and $P_{i}^{\prime \prime}(x)$ are continuous at each point $\left(u_{i}, v_{i}\right)$, where $i=0,1,2, \ldots, n$

(iv) $P_{i}\left(u_{i}\right)=v_{i}$, where $i=0,1,2, \ldots, n$

If $P_{i}^{\prime \prime}\left(u_{0}\right)=P_{i}^{\prime \prime}\left(u_{n}\right)=0$ and $P_{i}(u), P_{i}^{\prime}(u)$, and $P_{i}^{\prime \prime}(u)$ are all continuous in $\left(u_{0}, u_{n}\right)$, then this cubic spline is called as natural spline [20].

Many applications make use of slopes. So let us denote the cubic spline function that is obtained in terms of first derivatives to be $m_{i}$. The cubic spline $P(u)$ formula for an initial value problem in $u_{i-1} \leq u \leq u_{i}$ in terms of its first derivatives $P^{\prime}\left(u_{i}\right)=m_{i}$ can be obtained by using Hermite's interpolation formula as follows [21, 22]:

$$
\begin{aligned}
P(u)= & m_{i-1} \frac{\left(u_{i}-u\right)^{2}\left(u-u_{i-1}\right)}{h^{2}}-m_{i} \frac{\left(u-u_{i-1}\right)^{2}\left(u_{i}-u\right)}{h^{2}} \\
& +v_{i-1} \frac{\left(u_{i}-u\right)^{2}\left[2\left(u-u_{i-1}\right)+h\right]}{h^{3}} \\
& +v_{i} \frac{\left(u-u_{i-1}\right)^{2}\left[2\left(u_{i}-u\right)+h\right]}{h^{3}}
\end{aligned}
$$

where $h=u_{i}-u_{i-1}$ for all $i$ :

$$
\begin{aligned}
P^{\prime}(u)= & \frac{m_{i-1}}{h^{2}}\left(u_{i}-u\right)\left(2 u_{i-1}+u_{i}-3 u\right) \\
& -\frac{m_{i}}{h^{2}}\left(u-u_{i-1}\right)\left(u_{i-1}+2 u_{i}-3 u\right) \\
& +\frac{6}{h^{3}}\left(v_{i}-v_{i-1}\right)\left(u_{i}-u\right)\left(u-u_{i-1}\right), \\
P^{\prime \prime}(u)= & -2 \frac{m_{i-1}}{h^{2}}\left[u_{i-1}+2 u_{i}-3 u\right] \\
& -2 \frac{m_{i}}{h^{2}}\left[2 u_{i-1}+u_{i}-3 u\right] \\
& +\frac{6}{h^{3}}\left(v_{i}-v_{i-1}\right)\left[u_{i-1}+u_{i}-2 u\right] .
\end{aligned}
$$


Setting $u=u_{i}$ and $P\left(u_{i}\right)=v_{i}$ for all $i$ in (7), we have

$$
P^{\prime \prime}\left(u_{i}\right)=\frac{2 m_{i-1}}{h}+\frac{4 m_{i}}{h}-\frac{6}{h^{2}}\left(P_{i}-P_{i-1}\right) \text {. }
$$

Now consider a differential equation of first order with the initial condition as follows:

$$
\frac{\mathrm{d} v}{\mathrm{~d} u}=f(u, v) \text { and } v\left(u_{0}\right)=v_{0}
$$

On differentiating (9) twice with respect to $u$,

$$
v^{\prime \prime}(u)=f_{u}(u, v)+f_{v}(u, v) f(u, v) .
$$

Taking $u=u_{i}$ and $P\left(u_{i}\right)=v_{i}$, the above equation becomes

$$
P^{\prime \prime}\left(u_{i}\right)=f_{u}\left(u_{i}, P_{i}\right)+f_{v}\left(u_{i}, P_{i}\right) f\left(u_{i}, P_{i}\right) .
$$

On equating (8) and (11), we obtain

$$
\frac{2 m_{i-1}}{h}+\frac{4 m_{i}}{h}-\frac{6}{h^{2}}\left(P_{i}-P_{i-1}\right)=f_{u}\left(u_{i}, P_{i}\right)+f_{v}\left(u_{i}, P_{i}\right) f\left(u_{i}, P_{i}\right) .
$$

From this, we can compute $P_{i}$ 's. Substituting these $P_{i}$ 's in (5) gives the required solution. The convergence of this method has been proved by Patricio [21].

\section{Fuzzy Initial Value Problem}

Consider the first-order fuzzy differential equation as

$$
u^{\prime}(\xi)=f(\xi, u(\xi)), \quad \xi \in\left[\xi_{0}, T\right], T \geq 0,
$$

with the initial condition $u\left(\xi_{0}\right)=u_{0} \in \mathbb{F}$, where $u$ is a fuzzy function of the crisp variable $\xi$; that is, $u \in \mathbb{F}$, which is unknown. $f:\left[\xi_{0}, T\right] \times \mathbb{F} \longrightarrow \mathbb{F}$, which is a fuzzy function. $u^{\prime}$ is the fuzzy derivative of $u$, and $u\left(\xi_{0}\right)$ is a fuzzy number. Here, let us assume the fuzzy number to be a triangular fuzzy number.

For $\alpha \in[0,1]$, let us denote the $\alpha$-level sets:

$$
[u(\xi)]_{\alpha}=\left[[u(\xi)]_{l}(\alpha),[u(\xi)]_{r}(\alpha)\right] \text {, and }\left[u\left(\xi_{0}\right)\right]_{\alpha}=\left[\left[u\left(\xi_{0}\right)\right]_{l}(\alpha),\left[u\left(\xi_{0}\right)\right]_{r}(\alpha)\right] \text {. }
$$

Also,

$[f(\xi, u(\xi))]_{\alpha}=\left[f_{1}(\xi, u(\xi) ; \alpha), f_{2}(\xi, u(\xi) ; \alpha)\right]$, where

$$
\begin{aligned}
& f_{1}(\xi, u(\xi) ; \alpha)=\min \left\{f(\xi, s) \mid s \in\left[[u(\xi)]_{l}(\alpha),[u(\xi)]_{r}(\alpha)\right]\right\}=G\left(\xi,[u(\xi)]_{l}(\alpha),[u(\xi)]_{r}(\alpha)\right) \\
& f_{2}(\xi, u(\xi) ; \alpha)=\max \left\{f(\xi, s) \mid s \in\left[[u(\xi)]_{l}(\alpha),[u(\xi)]_{r}(\alpha)\right]\right\}=H\left(\xi,[u(\xi)]_{l}(\alpha),[u(\xi)]_{r}(\alpha)\right)
\end{aligned}
$$

The mapping $f:\left[\xi_{0}, T\right] \times \mathbb{F} \longrightarrow \mathbb{F}$ is a fuzzy process, and where the derivatives $f^{(i)} \in \mathbb{F}$, for $i=1,2, \ldots, p$, are defined as

$$
\left[f^{(i)}(\xi, u(\xi))\right]_{\alpha}=\left[f_{1}^{(i)}(\xi, u(\xi) ; \alpha), f_{2}^{(i)}(\xi, u(\xi) ; \alpha)\right]
$$

$$
\begin{aligned}
& f_{1}^{(i)}(\xi, u(\xi) ; \alpha)=\min \left\{f^{(i)}(\xi, s) \mid s \in\left[[u(\xi)]_{l}(\alpha),[u(\xi)]_{r}(\alpha)\right]\right\} \\
& f_{2}^{(i)}(\xi, u(\xi) ; \alpha)=\max \left\{f^{(i)}(\xi, s) \mid s \in\left[[u(\xi)]_{l}(\alpha),[u(\xi)]_{r}(\alpha)\right]\right\} .
\end{aligned}
$$

Equation (13) can be replaced by an equivalent system of equations, and hence,

$$
\left[u^{\prime}(\xi)\right](\alpha)=\left\{\left[u^{\prime}(\xi)\right]_{l}(\alpha),\left[u^{\prime}(\xi)\right]_{r}(\alpha)\right\}
$$


where

$$
\begin{aligned}
{\left[u^{\prime}(\xi)\right]_{l}(\alpha) } & =f_{1}(\xi, u(\xi) ; \alpha)=G\left(\xi,[u(\xi)]_{l}(\alpha),[u(\xi)]_{r}(\alpha)\right), \quad(\text { by }(15)), \\
{\left[u^{\prime}(\xi)\right]_{r}(\alpha) } & =f_{2}(\xi, u(\xi) ; \alpha)=H\left(\xi,[u(\xi)]_{l}(\alpha),[u(\xi)]_{r}(\alpha)\right), \quad(\text { by }(16)) .
\end{aligned}
$$

$$
\left[U\left(\xi_{n}\right)\right]_{\alpha}=\left[\left[U\left(\xi_{n}\right)\right]_{l}(\alpha),\left[U\left(\xi_{n}\right)\right]_{r}(\alpha)\right],
$$

The system of equations (20) and (21) will have a unique solution, $\left[[u(\xi)]_{l}(\alpha),[u(\xi)]_{r}(\alpha)\right] \in J=C\left(\left[\xi_{0}, \mathbb{F}\right]\right) \times C\left(\left[\xi_{0}\right.\right.$, $\mathbb{F}])$. Thus, given fuzzy differential equation (13) possesses a unique solution on $J$.

Usually, equations (20) and (21) can be solved analytically. Yet, in most of the cases, this becomes tedious, and hence, a numerical approach to these systems of equations has to be considered.

\section{Cubic Spline Method for Solving Fuzzy Initial Value Problem}

as the exact solution of (13):

$$
\left[P\left(\xi_{n}\right)\right]_{\alpha}=\left[\left[P\left(\xi_{n}\right)\right]_{l}(\alpha),\left[P\left(\xi_{n}\right)\right]_{r}(\alpha)\right],
$$

as the approximated solution of (13) at $\xi_{n}$ where $0 \leq n \leq N$.

Now let us calculate the solutions by mesh points at $\xi_{0}<\xi_{1}<\ldots<\xi_{N}=T, h=\left(T-\xi_{0}\right) / N$, and $\xi_{n}=\xi_{0}+n h$, where $n=0,1,2, \ldots, N$.

The cubic spline function $P(\xi ; \alpha)$ for a fuzzy initial value problem in $\xi_{i-1} \leq \xi \leq \xi_{i}$ in terms of its first derivatives $P^{\prime}\left(\xi_{i} ; \alpha\right)=m_{i}$ is given as

Assume that

$$
\begin{aligned}
P(\xi ; \alpha)=[P(\xi)]_{\alpha}= & m_{i-1} \frac{\left(\xi_{i}-\xi\right)^{2}\left(\xi-\xi_{i-1}\right)}{h^{2}}-m_{i} \frac{\left(\xi-\xi_{i-1}\right)^{2}\left(\xi_{i}-\xi\right)}{h^{2}} \\
& +\left([u(\xi)]_{i-1}(\alpha)\right) \frac{\left(\xi_{i}-\xi\right)^{2}\left[2\left(\xi-\xi_{i-1}\right)+h\right]}{h^{3}} \\
& +\left([u(\xi)]_{i}(\alpha)\right) \frac{\left(\xi-\xi_{i-1}\right)^{2}\left[2\left(\xi_{i}-\xi\right)+h\right]}{h^{3}},
\end{aligned}
$$

where $h=\xi_{i}-\xi_{i-1}$. But, we know that

$$
[P(\xi)]_{\alpha}=\left[[P(\xi)]_{l}(\alpha),[P(\xi)]_{r}(\alpha)\right],
$$

where

$$
\begin{aligned}
{[P(\xi)]_{l}(\alpha)=} & m_{i-1} \frac{\left(\xi_{i}-\xi\right)^{2}\left(\xi-\xi_{i-1}\right)}{h^{2}}-m_{i} \frac{\left(\xi-\xi_{i-1}\right)^{2}\left(\xi_{i}-\xi\right)}{h^{2}} \\
& +\left(\left[[u(\xi)]_{l}\right]_{i-1}(\alpha)\right) \frac{\left(\xi_{i}-\xi\right)^{2}\left[2\left(\xi-\xi_{i-1}\right)+h\right]}{h^{3}} \\
& +\left(\left[[u(\xi)]_{l}\right]_{i}(\alpha)\right) \frac{\left(\xi-\xi_{i-1}\right)^{2}\left[2\left(\xi_{i}-\xi\right)+h\right]}{h^{3}}, \\
{[P(\xi)]_{r}(\alpha)=} & m_{i-1} \frac{\left(\xi_{i}-\xi\right)^{2}\left(\xi-\xi_{i-1}\right)}{h^{2}}-m_{i} \frac{\left(\xi-\xi_{i-1}\right)^{2}\left(\xi_{i}-\xi\right)}{h^{2}} \\
& +\left(\left[[u(\xi)]_{r}\right]_{i-1}(\alpha)\right) \frac{\left(\xi_{i}-\xi\right)^{2}\left[2\left(\xi-\xi_{i-1}\right)+h\right]}{h^{3}} \\
& +\left(\left[[u(\xi)]_{r}\right]_{i}(\alpha)\right) \frac{\left(\xi-\xi_{i-1}\right)^{2}\left[2\left(\xi_{i}-\xi\right)+h\right]}{h^{3}} .
\end{aligned}
$$


By carrying out simple and similar calculations for (26) and (27) as given in "cubic spline function approximation for initial value problems" (especially equations from (6)-(12)), we obtain the following set of equations:

$$
\begin{gathered}
\frac{2 m_{i-1}}{h}+\frac{4 m_{i}}{h}-\frac{6}{h^{2}}\left(\left[P_{l}\right]_{i}-\left[P_{l}\right]_{i-1}\right)=G_{\xi}\left(\xi_{i},\left[P_{l}\right]_{i} ; \alpha\right)+G_{u(\xi)}\left(\xi_{i},\left[P_{l}\right]_{i} ; \alpha\right) G\left(\xi_{i},\left[P_{l}\right]_{i} ; \alpha\right), \\
\frac{2 m_{i-1}}{h}+\frac{4 m_{i}}{h}-\frac{6}{h^{2}}\left(\left[P_{r}\right]_{i}-\left[P_{r}\right]_{i-1}\right)=H_{\xi}\left(\xi_{i},\left[P_{r}\right]_{i} ; \alpha\right)+H_{u(\xi)}\left(\xi_{i},\left[P_{r}\right]_{i} ; \alpha\right) H\left(\xi_{i},\left[P_{r}\right]_{i} ; \alpha\right),
\end{gathered}
$$

where $i=1,2, \ldots, n$ and $h=\xi_{i}-\xi_{i-1}$. From (28), $\left[P_{l}\right]_{i}$ 's can be computed, and they are substituted in (26) to obtain the solution, $[P(\xi)]_{l}(\alpha)$. Similarly, $\left[P_{r}\right]_{i}$ 's can be evaluated from (29) and are substituted in (27) to yield $[P(\xi)]_{r}(\alpha)$. Each $P_{i}$ value depends on $P_{(i-1)}$ th value, for $i=1,2, \ldots, n$.

Both these solutions collectively yield the desired solution $[P(\xi)]_{\alpha}$ of $(13)$ at a fixed $\xi \in\left[\xi_{i-1}, \xi_{i}\right], i=1,2, \ldots, n$.

4.1. Convergence of Fuzzy Cubic Spline Method. Let us consider the equations:

$$
\begin{aligned}
{\left[P^{\prime \prime}\left(\xi_{i}\right)\right]_{l}(\alpha)=} & \frac{2 m_{i-1}}{h}+\frac{4 m_{i}}{h}-\frac{6}{h^{2}}\left(\left[P_{l}\right]_{i}-\left[P_{l}\right]_{i-1}\right) \\
{\left[u^{\prime \prime}\left(\xi_{i}\right)\right]_{l}(\alpha)=} & G_{\xi}\left(\xi_{i},\left[P_{l}\right]_{i} ; \alpha\right) \\
& +G_{u(\xi)}\left(\xi_{i},\left[P_{l}\right]_{i} ; \alpha\right) G\left(\xi_{i},\left[P_{l}\right]_{i} ; \alpha\right) .
\end{aligned}
$$

According to the results given in the work by Ahlberg et al. [19] (p. 34) and Theorem 1 , if $G(\xi, u(\xi) ; \alpha) \in C^{3}\left[\xi_{0}, T\right]$, we have

$$
\left|u^{(p)}(\xi)-P^{(p)}(\xi)\right|=O(h)^{4-p}, \quad p=0,1,2 \text {, and } 3 .
$$

If $p=2$, then the above equation can be written as

$$
\left|u^{\prime \prime}(\xi)-P^{\prime \prime}(\xi)\right|=O(h)^{2} .
$$

At $\xi=\xi_{i}$, for $i=1,2, \ldots, n$, we have

$$
\begin{aligned}
& \left|u^{\prime \prime}\left(\xi_{i}\right)-P^{\prime \prime}\left(\xi_{i}\right)\right|=O(h)^{2}, \text { where } h=\max _{i} h_{i} \\
& \text { (or) }\left[u^{\prime \prime}\left(\xi_{i}\right)\right]_{l}(\alpha)=O\left(h^{2}\right)+\left[P^{\prime \prime}\left(\xi_{i}\right)\right]_{l}(\alpha) \text {, } \\
& \Rightarrow h^{2}\left[u^{\prime \prime}\left(\xi_{i}\right)\right]_{l}(\alpha)=2 h m_{i-1}+4 h m_{i}-6\left(\left[P_{l}\right]_{i}-\left[P_{l}\right]_{i-1}\right)+O\left(h^{4}\right) \text {. } \\
& r\left(\left[P_{l}\right]_{i}\right)=O\left(h^{4}\right) \text {. }
\end{aligned}
$$

Again, with (31), we obtain $\left[P_{l}\right]_{i}$ explicitly or not according to the linearity or nonlinearity of $G(\xi, u(\xi) ; \alpha)$ in $u(\xi)$. Then, we can write

$$
\left[P_{l}\right]_{i}=c_{1}+c_{2} \alpha+O\left(h^{4}\right)
$$

Hence, the order of the method is sustained, and it is true for $\xi \in\left[\xi_{0}, T\right]$.

From (26), we have

where $c_{1}$ and $c_{2}$ are constants and $\alpha \in[0,1]$ or

$$
\left|[P(\xi)]_{l}(\alpha)\right|<\left|m_{j-1} h-m_{j} h+\left(\left[[u(\xi)]_{l}\right]_{j-1}(\alpha)\right)+\left(\left[[u(\xi)]_{l}\right]_{j}(\alpha)\right)\right|
$$

where $\xi_{j-1} \leq \xi \leq \xi_{j}, \forall j=0,1, \ldots, n$.

Similarly, by considering the equations,

$$
\begin{aligned}
& {\left[P^{\prime \prime}\left(\xi_{i}\right)\right]_{r}(\alpha)=\frac{2 m_{i-1}}{h}+\frac{4 m_{i}}{h}-\frac{6}{h^{2}}\left(\left[P_{r}\right]_{i}-\left[P_{r}\right]_{i-1}\right),} \\
& {\left[u^{\prime \prime}\left(\xi_{i}\right)\right]_{r}(\alpha)=H_{\xi}\left(\xi_{i},\left[P_{r}\right]_{i} ; \alpha\right)+H_{u(\xi)}\left(\xi_{i},\left[P_{r}\right]_{i} ; \alpha\right) H\left(\xi_{i},\left[P_{r}\right]_{i} ; \alpha\right),}
\end{aligned}
$$


we get

$$
\left|[P(\xi)]_{r}(\alpha)\right|<\left|m_{j-1} h-m_{j} h+\left(\left[[u(\xi)]_{r}\right]_{j-1}(\alpha)\right)+\left(\left[[u(\xi)]_{r}\right]_{j}(\alpha)\right)\right|
$$

Thus, from (24), we obtain

$$
\left|[P(\xi)]_{\alpha}\right|<\left|m_{j-1} h-m_{j} h+[u(\xi)]_{j-1}(\alpha)+[u(\xi)]_{j}(\alpha)\right|, \xi \in\left[\xi_{j-1}, \xi_{j}\right], \quad \forall j=0,1, \ldots, n .
$$

\section{Numerical Illustration (Exponential Decay Problem with Decay Constant as 1)}

Consider the fuzzy differential equation

$$
y^{\prime}(\xi)=-y(\xi)
$$

with $y(0)=(0.5,1,1.5)$ as its fuzzy initial condition. Let us find the solution of (41) at $\xi=0.2$ and 0.3 .

Equation (41) can be modified into a system of ordinary differential equations as follows:

$$
\begin{gathered}
{\left[y^{\prime}(\xi)\right]_{l}(\alpha)=-[y(\xi)]_{l}(\alpha),[y(0)]_{l}(\alpha)=0.5 \alpha+0.5,} \\
{\left[y^{\prime}(\xi)\right]_{r}(\alpha)=-[y(\xi)]_{r}(\alpha),[y(0)]_{r}(\alpha)=1.5-0.5 \alpha .}
\end{gathered}
$$

The solution of these two equations collectively gives the solution of (41). Therefore, the exact solution of (41) is

$$
\begin{aligned}
{[Y(\xi)](\alpha) } & =[[Y l(\xi)](\alpha),[\operatorname{Yr}(\xi)](\alpha)] \\
& =[(0.5 \alpha+0.5) \exp (-\xi),(1.5-0.5 \alpha) \exp (-\xi)]
\end{aligned}
$$

Now let us compute the numerical solution of (41) by using the cubic spline method.

For simplicity, assume $h=0.1$.

Consider equation $(42)$, here $G(\xi, y ; \alpha)=-y$ and so $G_{\xi}(\xi, y ; \alpha)=0$ and $G_{y}(\xi, y ; \alpha)=-1$.

Also, $G\left(\xi_{i},\left[P_{l}\right]_{i} ; \alpha\right)=-\left[P_{l}\right]_{i}$.

Using (28) at $i=1$ and $\xi=0.1$, we get

$$
20 m_{0}+40 m_{1}-600\left(\left[P_{l}\right]_{1}-\left[P_{l}\right]_{0}\right)=\left[P_{l}\right]_{1} \text {. }
$$

Since $m_{i}=\left[P_{l}\right]^{\prime}\left(\xi_{i}\right), m_{0}=-0.5 \alpha-0.5$ and $m_{1}=-\left[P_{l}\right]_{1}$, the above equation on simplification gives

$$
\left[P_{l}\right]_{1}=\frac{290 \alpha+290}{641}
$$

Similarly, at $i=2$ and $\xi=0.2$, (28) becomes

$$
\left[P_{l}\right]_{2}=\frac{1}{641^{2}}(168200 \alpha+168200) \text {. }
$$

This is the approximate solution of (42) at $\xi=0.2$. By using (42) at $i=3$, we obtain

$$
20 m_{2}+40 m_{3}-600\left(\left[P_{l}\right]_{3}-\left[P_{l}\right]_{2}\right)=\left[P_{l}\right]_{3},
$$

where $m_{2}=-\left[P_{l}\right]_{2}$ and $m_{3}=-\left[P_{l}\right]_{3}$. This equation gives the approximate solution of (42) at $\xi=0.3$.

Now consider equation (43), here $H(\xi, y ; \alpha)=-y$, and hence, $\quad H_{\xi}(\xi, y ; \alpha)=0, \quad H_{y}(\xi, y ; \alpha)=-1, \quad$ and $H\left(\xi_{i},\left[P_{r}\right]_{i} ; \alpha\right)=-P_{i}$.

By using (29) at $i=1$ and $\xi=0.1$, we obtain

$$
\left[P_{r}\right]_{1}=\frac{-290 \alpha+870}{641}
$$

where $m_{i}=\left[P_{r}\right]^{\prime}\left(\xi_{i}\right)$, for all $i$.

From (29), taking $i=2$ and $\xi=0.2$, we have

$$
20 m_{1}+40 m_{2}-600\left(\left[P_{r}\right]_{2}-\left[P_{r}\right]_{1}\right)=\left[P_{r}\right]_{2},
$$

$\therefore\left[P_{r}\right]_{2}=\frac{1}{641^{2}}(-168200 \alpha+504600)$ (on simplification).

Similarly, for $i=3$ and $\xi=0.3$ in (29), we get

$$
20 m_{2}+40 m_{3}-600\left(\left[P_{r}\right]_{3}-\left[P_{r}\right]_{2}\right)=\left[P_{r}\right]_{3},
$$

where $m_{2}=-\left[P_{r}\right]_{2}$ which is given by (50) and $m_{3}=-\left[P_{r}\right]_{3}$. This equation on further simplification gives the approximate solution of (43) at $\xi=0.3$.

Tables 1 and 2 represent the comparison of the solutions for equation (41) that are obtained by exact, cubic spline method and Taylor's method of order, $p=2$ at $\xi=0.2$ with $h=0.1$. Comparison of exact and cubic spline solutions at $\xi=0.2$ is graphically given in Figure 1 . Similarly, Figure 2 interprets the compared results of exact and cubic spline at $\xi=0.3$ of step length $h=0.1$.

In general, the numerical solution of the fuzzy differential equation by using the cubic spline method can be given as

$$
\left[P_{i}\right]_{\alpha}=\left[\left[P_{l}\right]_{i}(\alpha),\left[P_{r}\right]_{i}(\alpha)\right]
$$

where $i=1,2, \ldots, n$, i.e., $[P(\xi)](\alpha)=[[P l(\xi)](\alpha),[\operatorname{Pr}(\xi)]$ $(\alpha)$ ], for a fixed $\xi$. 
TABLE 1: Comparison of the results (approximated to 9 decimals) obtained by exact, cubic spline method and Taylor's method of order, $p=2$ at $\xi=0.2$ with $h=0.1$ for equation (42).

\begin{tabular}{lccc}
\hline$\alpha$-cut & $\begin{array}{c}\text { Exact solution } \\
\text { - }\end{array}$ & $\begin{array}{c}\text { Cubic } \\
\text { spline } P \text { l }\end{array}$ & $\begin{array}{c}\text { Taylor solution } \\
T 1 \text { for } p=2\end{array}$ \\
\hline 0.0 & 0.409365377 & 0.409364268 & 0.409512500 \\
0.1 & 0.450301914 & 0.450300695 & 0.450463750 \\
0.2 & 0.491238452 & 0.491237122 & 0.491415000 \\
0.3 & 0.532174990 & 0.532173549 & 0.532366250 \\
0.4 & 0.573111527 & 0.573109976 & 0.573317500 \\
0.5 & 0.614048065 & 0.614046403 & 0.614268750 \\
0.6 & 0.654984602 & 0.654982830 & 0.655220000 \\
0.7 & 0.695921140 & 0.695919256 & 0.696171250 \\
0.8 & 0.736857678 & 0.736855683 & 0.737122500 \\
0.9 & 0.777794215 & 0.777792110 & 0.778073750 \\
1.0 & 0.818730753 & 0.818728537 & 0.819025000 \\
\hline
\end{tabular}

TABLE 2: Comparison of the results (approximated to 9 decimals) obtained by exact, cubic spline method and Taylor's method of order, $p=2$ at $\xi=0.2$ with $h=0.1$ for equation (43).

\begin{tabular}{|c|c|c|c|}
\hline$\alpha$-cut & Exact solution $Y r$ & Cubic spline $\mathrm{Pr}$ & Taylor solution $\operatorname{Tr}$ for $p=2$ \\
\hline 0.0 & 1.228096130 & 1.228092805 & 1.228537500 \\
\hline 0.1 & 1.187159592 & 1.187156379 & 1.187586250 \\
\hline 0.2 & 1.146223054 & 1.146219952 & 1.146635000 \\
\hline 0.3 & 1.105286517 & 1.105283525 & 1.105683750 \\
\hline 0.4 & 1.064349979 & 1.064347098 & 1.064732500 \\
\hline 0.5 & 1.023413441 & 1.023410671 & 1.023781250 \\
\hline 0.6 & 0.982476904 & 0.982474244 & 0.982830000 \\
\hline 0.7 & 0.941540366 & 0.941537818 & 0.941878750 \\
\hline 0.8 & 0.900603828 & 0.900601391 & 0.900927500 \\
\hline 0.9 & 0.859667291 & 0.859664964 & 0.859976250 \\
\hline 1.0 & 0.818730753 & 0.818728537 & 0.819025000 \\
\hline
\end{tabular}

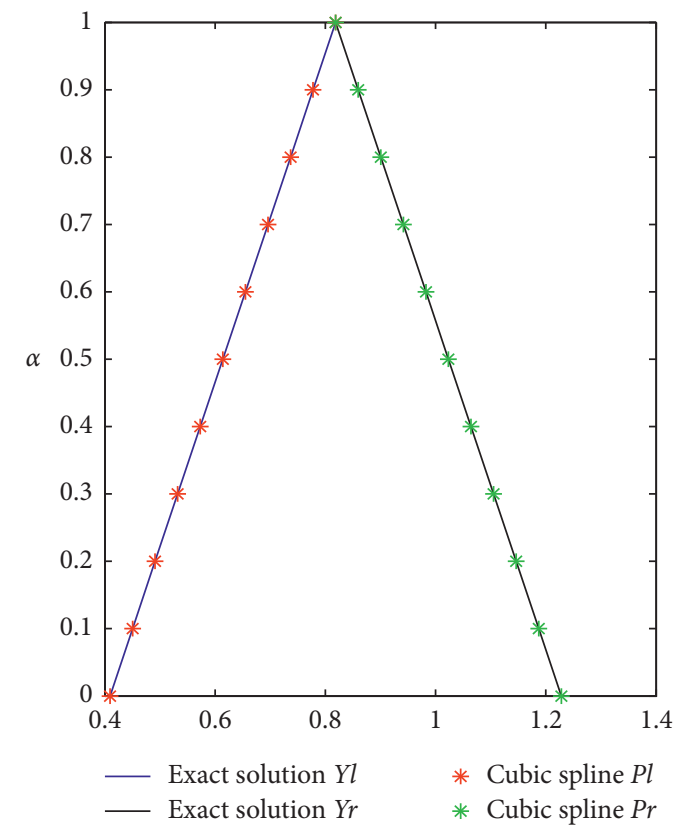

FIgURE 1: Comparison of exact and cubic spline solutions at $\xi=0.2$. 


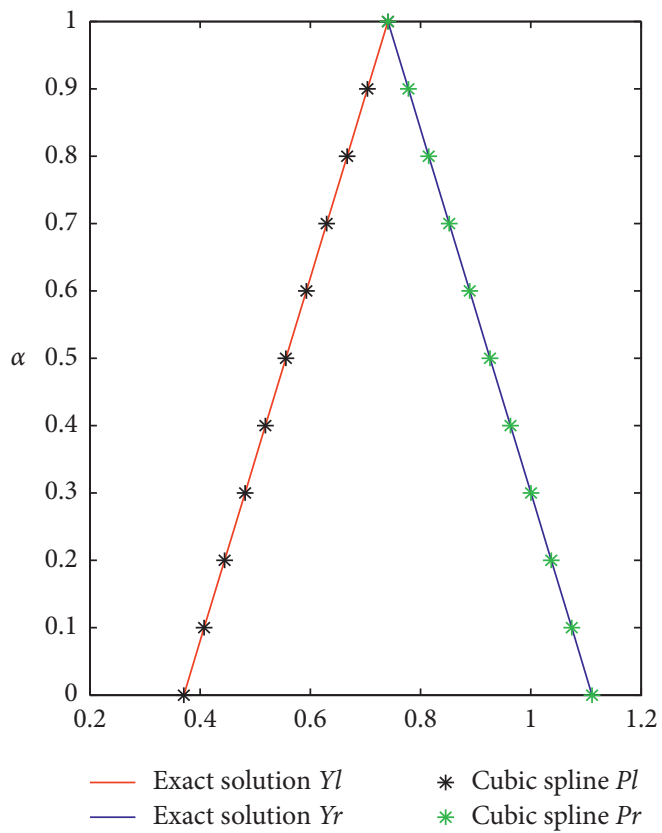

Figure 2: Comparison of exact and cubic spline solutions at $\xi=0.3$ for $h=0.1$.

\section{Conclusion}

In this article, a new class of cubic spline function method is introduced for solving fuzzy differential equations subject to fuzzy initial conditions. The desired solution which is obtained is of $O\left(h^{4}\right)$ convergence based on certain conditions on the derivatives. This numerical method is verified with an example, and the results are compared with the exact as well as with the solution obtained by Taylor's method of order, $p=2$. From the comparison of results, one can conclude that the proposed method is a single-step method that converges faster and has greater accuracy than the Taylor method of order two. In future, one can extend this method to solve higher-order linear and nonlinear fuzzy initial value problems.

\section{Data Availability}

No data were used to support the findings of the study.

\section{Conflicts of Interest}

The authors declare that they have no conflicts of interest.

\section{Authors' Contributions}

All authors contributed equally to this work. And, all the authors have read and approved the final version manuscript.

\section{Acknowledgments}

This research was funded by the Deanship of Scientific Research at Princess Nourah Bint Abdulrahman University through the Fast-Track Research Funding Program.

\section{References}

[1] S. N. Sivanandam, S. Sumathi, and S. N. Deepa, Introduction to Fuzzy Logic Using MATLAB, Springer, Berlin Heidelberg, 2007.

[2] L. A. Zadeh, "Fuzzy sets," Information and Control, vol. 8, no. 3, pp. 338-353, 1965

[3] S. S. L. Chang and L. A. Zadeh, "On fuzzy mapping and control," IEEE Transactions on Systems, Man, and Cybernetics, vol. SMC-2, no. 1, pp. 30-34, 1972.

[4] A. Kandel and W. J. Byatt, "Fuzzy differential equations," in Proceedings of the International Conference Cybernetics and Society, pp. 1213-1216, Tokyo-Kyoto, Japan, November 1978.

[5] B. Bede, "Fuzzy differential equations," Mathematics of Fuzzy Sets and Fuzzy Logic, vol. 295, pp. 171-191, 2013.

[6] O. Kaleva, "The cauchy problem for fuzzy differential equations," Fuzzy Sets and Systems, vol. 35, no. 3, pp. 389-396, 1990.

[7] S. Seikkala, "On the fuzzy initial value problem," Fuzzy Sets and Systems, vol. 24, no. 3, pp. 319-330, 1987.

[8] S. Song and C. Wu, "Existence and uniqueness of solutions to Cauchy problem of fuzzy differential equations," Fuzzy Sets and Systems, vol. 110, no. 1, pp. 55-67, 2000.

[9] J. J. Buckley and T. Feuring, "Fuzzy differential equations," Fuzzy Sets and Systems, vol. 110, no. 1, pp. 43-54, 2000.

[10] D. O’Regan, V. Lakshmikantham, and J. Nieto, "Initial and boundary value problems for fuzzy differential equations," Nonlinear Analysis: Theory, Methods \& Applications, vol. 54, no. 3, pp. 405-415, 2003.

[11] S. Melliani and L. S. Chadli, "Intuitionistic fuzzy differential equation," Notes on Intuitionistic Fuzzy Sets, vol. 6, no. 2, pp. 37-41, 2000.

[12] S. Chakraverty, S. Tapaswini, and D. Behera, Fuzzy Differential Equations and Applications for Engineers and Scientists, CRC Press, Taylor \& Francis Group, Boca Raton, FL, USA, 2017. 
[13] M. Ahamed and M. Hasan, "A new fuzzy version of Euler's method for solving differential equations with fuzzy initial values," Sians Malaysiana, vol. 40, pp. 651-657, 2011.

[14] S. Abbasbandy and T. A. Viranloo, "Numerical solutions of fuzzy differential equations by Taylor method," Computational Methods in Applied Mathematics, vol. 2, no. 2, pp. 113-124, 2002.

[15] T. Allahviranloo, N. Ahmady, and E. Ahmady, "Numerical solution of fuzzy differential equations by predictor-corrector method," Information Sciences, vol. 177, no. 7, pp. 1633-1647, 2007.

[16] R. Ezzati, K. Maleknejad, S. Khezerloo, and M. Khezerloo, "Convergence, consistency and stability in fuzzy differential equations," Iranian Journal of Fuzzy Systems, vol. 12, no. 7, pp. 95-112, 2015.

[17] L. T. Gomes, L. Carvalho de Barros, and B. Bede, Fuzzy Differential Equations in Various Approaches, Springer, New York, NY, USA, 2015.

[18] O. Kaleva, "A note on fuzzy differential equations," Nonlinear Analysis: Theory, Methods \& Applications, vol. 64, no. 5, pp. 895-900, 2006.

[19] J. H. Ahlberg, E. N. Nilson, and J. L. Walsh, The Theory of Splines and Their Applications, Academic Press INC., London, UK, 1967.

[20] P. Kandaswamy, K. Thilagavathy, and K. Gunavathi, Numerical Methods, S. Chand \& Company Pvt.Ltd, New Delhi, India, 2008.

[21] F. Patrício, "Cubic spline functions and initial value problems,” BIT, vol. 18, no. 3, pp. 342-347, 1978.

[22] S. S. Sastry, Introductory Methods of Numerical Analysis, PHI Learning Private Limited, New Delhi, India, 5th edition, 2012. 